\title{
Gapped Ferromagnetic Graphene Nanoribbons
}

\author{
D. Hou, ${ }^{1,2}$ J. H. Wei, ${ }^{2, *}$ and S. J. Xie ${ }^{1, \dagger}$ \\ ${ }^{1}$ School of Physics, National Key Laboratory of Crystal Materials, Shandong University, Jinan 250100, China \\ ${ }^{2}$ Department of Physics, Renmin University of China, Beijing 100872, China
}

(Dated: October 17, 2018)

\begin{abstract}
We theoretically design a graphene-based all-organic ferromagnetic semiconductor by terminating zigzag graphene nanoribbons (ZGNRs) with organic magnets. A large spin-split gap with $100 \%$ spin polarized density of states near the Fermi energy is obtained, which is of potential application in spin transistors. The interplays among electron, spin and lattice degrees of freedom are studied using the first-principles calculations combined with fundamental model analysis. All of the calculations consistently demonstrate that although no $d$ electrons existing, the antiferromagnetic $\pi-\pi$ exchange together with the strong spin-lattice interactions between organic magnets and ZGNRs make the ground state ferromagnetic. The fundamental physics makes it possible to optimally select the organic magnets towards practical applications.
\end{abstract}

PACS numbers: 73.22.Pr, 75.75.-c, 71.15.Mb

Introduction.-Since the experimental discovery of graphene in 2004 [1], graphene-based transistors have rapidly developed and are considered good candidates for post-silicon electronics [2]. However, they encounter an outstanding challenge of how to open a sizable and well-defined band gap in graphene to control the ONand OFF-states of the transistors. One realistic solution is constraining large-area graphene in one dimension to form graphene nanoribbons (GNRs) which have band gaps approximately inversely proportional to their widths [3]. In recent years, this scheme has been experimentally proved rather practical and thus motivated a new frontier area called "graphene nanoribbon electronics" [4 6].

Besides the gapped semiconductor properties suitable for electronics, graphene nanoribbons possess fascinating magnetic properties for spintronics. Zigzag graphene nanoribbons (ZGNRs) have spatial spin-polarized ground states with spin moments coupled ferromagnetically (FM) on the same edge and antiferromagnetically (AFM) between different edges [7, 8], which makes them potential materials for carbon-based spintronic devices avoiding heavy magnetic atoms. By means of electric field control, Boron/Nitron doping or edge termination, ZGNRs can exhibit half-metallicity that may be used for spin injection and filtration in metallic spintronics 9 - 12 .

On the other side, semiconductor spintronics has been the subject of many recent studies [13]. The central issue is how to develop spin transistors with appropriate ferromagnetic semiconductor materials for non-volatile memory applications [14]. By virtue of the unique electronic and magnetic properties mentioned above, GNRs may be ideal materials for spin transistors. However, the difficulty is that the ferromagnetic state of ZGNRs is not the ground state, and even worse for transistors, it is a gapless state. Therefore, the organic integration of electronic and magnetic properties of graphene for spin transistor is still an open question.

In this paper, we design a graphene nanoribbon ter- minated with organic magnets to give a possible solution for the above issue. In order to convert the ground state of ZGNRs from the AFM state to a gapped FM one, we use edge passivation of graphene nanoribbons by organic ferromagnetic radicals (one kind of organic magnets, see Fig.11). The physics is that the magnetic order of the host material will be changed when it exchangecouples to the localized spin of attached organic magnets [15]. Comparing with ZGNRs adsorbing magnetic transition metal atoms on their surfaces in literatures [16, 17], these carbon-based materials have the advantages of much weaker spin-orbit and hyperfine interactions, which should induce much longer spin coherent length. Furthermore, owing to the planar structure of the selected organic radical, above mentioned exchange coupling is tunable by manually rotating the radical plane to affect the spin polarization near the Fermi energy. Our results suggest that the controllable graphene-based spin transistors can be expected.

Methods.-Towards the practical applications, we firstly choose trimethylenemethane (TMM) as the organic magnet, for the reason that it has been widely investigated and designed for kinds of possible spintronic applications due to its high magnetic moments [18]. However, as will be demonstrated later, the dependence of the physics on this specific choice is quite weak. TMM contains 4 carbon and 6 hydrogen atoms with $\pi$-conjugated structure and has a ground state of triplet biradical, as shown in Fig 1b. Two unpaired electrons in TMM provide $2 \mu_{B}$ spin moments in total distributing via molecular orbital over all the carbon atoms. At its spin triplet ground states, three outer carbon atoms have the same kind of net spin density, while the inner one has the opposite (see Fig 1d). When attached to one of the ZGNRs' edges already possessing spin moments, TMM will strongly modify the properties of ZGNRs.

6-ZGNR terminated by hydrogen atoms is used as pristine ZGNR as shown in Fig 1a. The unit cell of 6-ZGNR 
a)

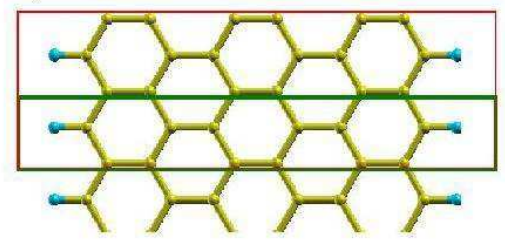

c)

6-ZGNR

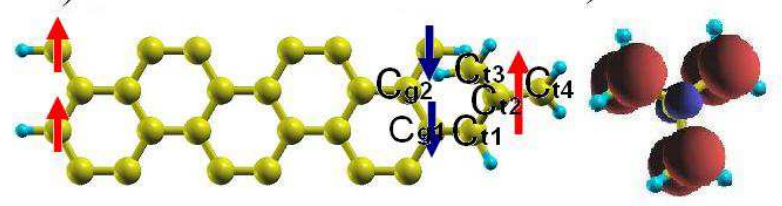

+-+ spin configuration b)

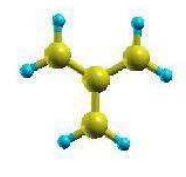

TMM

d)

Net spin density
Figure 1: (color online) Top view structures of (a) pristine 6-ZGNR, (b) TMM, (c) TMM terminated 6-ZGNRs, and (d) the net spin density of TMM. The yellow balls are carbon atoms and the cyanic ones are hydrogen atoms. The red/blue arrow indicates the up/down spin component.

contains 12 carbon atoms and 2 hydrogen atoms (see the figure inside the green rectangle in Fig (19). The length of periodic direction of the unit cell is $2.46 \AA$ (experiment value), while the repeated images of the molecular are separated by vacuum more than $8 \AA$ thick along the other two directions. To avoid direct interaction between images of terminated TMM, the zigzag direction of the unit cell is doubled (see the region inside the red rectangle in Fig 1a). In this supercell, TMM replaces one of the four hydrogen atoms, making the system terminated by TMM and hydrogen alternately on one edge (e.g. the right edge). The top view of one possible relaxed structure (that will be proved the ground state) is shown in Fig 11. Please be noted that the TMM biradical is not coplanar with ZGNR after atomic optimization.

The first-principles calculations are performed using Density Functional Theory (DFT) method implemented with SIESTA code 19] using the Perdew-Burke-Ernzerhof exchange-correlation functional[20]. Double- $\zeta$ plus polarization function (DZP) basis set is used, together with a mesh cutoff of $200 \mathrm{Ry}$ and norm-conserving pseudopotentials. All the atoms in the supercell are fully relaxed to fulfill the energy and force convergence of $10^{-5} \mathrm{eV}$ and $0.01 \mathrm{eV} / \AA$ respectively. The optimized structure parameters with different initial spin configurations are crosschecked using a plane-wave method implemented in Quantum Espresso code 21], with the same convergence criteria and a plane wave kinetic energy cutoff of 30 Ry. The identical results ensure the validity of the simulation parameters used, and other data are handled using SIESTA code solely.

Results. - In what follows, we use "++" and "+-" to re- spectively denote the FM and AFM spin configurations, where the first symbol denotes the left edge spin moment while the second denotes the right one of the graphene ribbon. Without losing generality, we designate "+/-" to represent up/down spin in what follows. The convergence of the simulation parameters is firstly checked by electronic property calculations of a pristine 6 -ZGNR with a $1 \times 1 \times 2$ supercell. The "+-" order is confirmed to be the ground state with a net spin moments of $0.26 \mu_{B}$ localized on each edge atom, and its total energy is 26 $\mathrm{meV} /$ supercell lower than the "++" one. These values are consistent with previously reported results and used as reference data [7, 8].

The TMM units are then attached to the right side of the ZGNR. Since the spin moments on TMM and ZGNRs can align either parallel or anti-parallel, the "++" and "+-" spin configurations will extend to four possible ones: "+-+", "++-","+--" and "+++", with the rightmost symbol representing the net spin moments on TMM. Full atomic relaxation calculations are done separately using these four possible spin configurations to find the ground state. It is found that both the bond length and the dihedral angle between the ZGNR and TMM are varied with different spin configurations ( we will discuss this point in details later). The relative total energy ( taking $E_{+-+}$as reference), converged net spin moments and optimized bond structures are summarized in Tab! Crosschecking with Quantum Espresso presents highly consistent results, as shown in Tab!

As presented in Tab凹, the "+-+" state is the ground state. It has a total energy of $36 \mathrm{meV}$ lower than that of "++-" state, $578 \mathrm{meV}$ than that of "+--" state and $612 \mathrm{meV}$ than that of "+++" state, respectively. These data indicate that, in the ground state of the radical terminated graphene nanoribbon, the net spin moments of TMM radical antiferromagnetically couples to the nearest edge spin of ZGNR. As the energy difference between pristine "+-" "++" ZGNR is $26 \mathrm{meV}$, the present coupling is much stronger than the interedge superexchange of ZGNR[22]. A relative result was reported just recently by Atodiresei et al. focusing on the spin polarization of nonmagnetic benzene, cyclopentadienyl radical and cyclooctatetraene molecule adsorbed onto a ferromagnetic $2 \mathrm{ML} \mathrm{Fe} / \mathrm{W}(110)$ surface. It is found that at the organic molecule site an inversion of the spin polarization occurs with respect to the ferromagnetic surface, resulting from the antiferromagnetic coupling between $\pi$-electrons of the molecule and the $d$-electrons of Fe atoms [23]. While in the present case, the strong antiferromagnetic coupling presented here results from the $\pi-\pi$ interactions.

Comparing the corresponding structures of the four spin configurations manifests the close connection between the spin coupling and the variation of the ZGNRTMM bond length (or dihedral angle). It can be seen from Tab! that the changes of bond lengths and spin moments among the four possible spin configurations 
Table I: Relative total energy, converged net spin moments on selected carbon atoms, optimized bond lengths and spin angles for all possible spin configurations of TMM terminated 6ZGNR. The meanings of the symbols for the carbon atoms are depicted in Fig[1;. The values in brackets are corresponding results obtained by Quantum Espresso code.

\begin{tabular}{|c|c|c|c|c|c|c|c|c|c|c|c|c|}
\hline \multirow{2}{*}{ Label } & \multirow{2}{*}{${\mathrm{E}-\mathrm{E}_{+-+}}(\mathrm{meV})$} & \multicolumn{9}{|c|}{ Net Spin Moments $\left(\mu_{B}\right)$} & \multicolumn{5}{c|}{ Bond Lengths $(\AA)$} & Spin Angle \\
\cline { 3 - 15 } & & $\mathrm{C}_{g 1}$ & $\mathrm{C}_{g 2}$ & $\mathrm{C}_{t 1}$ & $\mathrm{C}_{t 2}$ & $\mathrm{C}_{t 3}$ & $\mathrm{C}_{t 4}$ & $\mathrm{C}_{g 1}-\mathrm{C}_{t 1}$ & $\mathrm{C}_{t 1}-\mathrm{C}_{t 2}$ & $\mathrm{C}_{t 2}-\mathrm{C}_{t 3}$ & $\mathrm{C}_{t 2}-\mathrm{C}_{t 4}$ & $\mathrm{C}_{g 1}-\mathrm{C}_{t 1}$ \\
\hline+-+ & 0 & -0.08 & 0.09 & 0.25 & -0.11 & 0.60 & 0.51 & $1.39(1.38)$ & $1.48(1.48)$ & $1.42(1.41)$ & $1.40(1.40)$ & $180^{\circ}$ \\
\hline++- & 36 & 0.08 & -0.08 & -0.24 & 0.11 & -0.60 & -0.51 & $1.39(1.38)$ & $1.48(1.48)$ & $1.42(1.41)$ & $1.40(1.40)$ & $180^{\circ}$ \\
\hline+-- & 578 & -0.24 & 0.02 & -0.63 & 0.11 & -0.68 & -0.68 & $1.48(1.48)$ & $1.43(1.43)$ & $1.42(1.42)$ & $1.42(1.42)$ & $70^{\circ}$ \\
\hline+++ & 612 & 0.23 & -0.02 & 0.63 & -0.11 & 0.68 & 0.68 & $1.48(1.48)$ & $1.43(1.43)$ & $1.42(1.42)$ & $1.42(1.42)$ & $70^{\circ}$ \\
\hline
\end{tabular}

mainly take place around the $\mathrm{C}_{g 1}-\mathrm{C}_{t 1}$ bond. When TMM and ZGNR are coupled antiferromagnetically ("+-+" and "++-" states), the length of the $\mathrm{C}_{g 1}-\mathrm{C}_{t 1}$ bond is shortened about $0.1 \AA$ relative to those of "+--" and "+++" states. It is reasonable to attribute such a large distortion to the softness of the all-organic system and the strong spin-lattice interaction. It is further found that, if the dihedral angle is disturbed, the bond length will changes correspondingly. As the $\pi$ electron orbital trends to be perpendicular to the $\sigma$ bonds surface, the dihedral angle reflects the spin angle of $\pi-\pi$ electrons on $\mathrm{C}_{g 1}$ and $\mathrm{C}_{t 1}$. Therefore, the calculation reveals that the $\pi-\pi$ spin coupling depends not only on the bond length $d$ but also on the spin angle $\theta$. We thus propose a spin-lattice (spinphonon) coupling model to illustrate the physics as follows,

$$
H_{\mathrm{T}}=H_{c}+J_{0}(1-\alpha u) \vec{S}_{g 1} \cdot \vec{S}_{t 1}+\frac{k}{2} u^{2}
$$

where $H_{c}$ denotes the invariant part (except the spin-spin coupling between $\mathrm{C}_{g 1}$ and $\mathrm{C}_{t 1}$ ) before and after spinlattice interaction involved. The second term is the $\pi$ $\pi$ spin interaction modulated by the bond distortion $u$ between $\mathrm{C}_{g 1}$ and $\mathrm{C}_{t 1}$, where $J_{0}$ is the coupling constant at $u=0, \alpha$ the spin-lattice coupling constant $(\alpha>0)$. The third one is the elastic potential energy caused by the bond distortion. As the magnetism of the terminated radical is robust, we take a classic approach with

$$
\vec{S}_{g 1} \cdot \vec{S}_{t 1}=S_{g 1} S_{t 1} \cos \theta
$$

By taking the differential of the Hamiltonian (1) with respect to the bond distortion $u$, we can obtain the stable state under certain fixed angle $\theta$,

$$
u=\frac{\alpha J_{0} S_{g 1} S_{t 1} \cos \theta}{k}=u_{0} \cos \theta
$$

which indicates that the optimal bond distortion $u$ will change with the spin angle $\theta$. We then obtain the optimal spin angel $\theta$ and bond distortion $u$ by minimizing the total energy, which is given as

$$
\left\{\begin{array}{l}
\theta=\pi \\
u=-u_{0}<0
\end{array}\right.
$$

It derives that the ground state is characterized by antiferromagnetic exchange interaction and a shorter $\mathrm{C}_{g 1^{-}}$ $\mathrm{C}_{t 1}$ bond length, which is consistent with our firstprinciples calculations as shown in Tab. I.

In order to elucidate the robust physics of the spinlattice coupling, we substitute the TMM radicals with $\mathrm{CH}_{2}$, the simplest carbon based organic radical holding the $s p^{2}$ hybridization. In the first principle calculation, the optimal bond length $d$ between $\mathrm{C}_{g 1}$ and $\mathrm{C}_{t 1}$ for each $\theta$ is obtained by minimizing the total energy. The calculated $d-\theta$ relation is shown in Fig. 2, which is well fitted with a cosine type line as deduced from our model. Therefore, in our proposed all-organic ferromagnetic graphene structure, the ferromagnetism is provided by the $\pi-\pi$ spin interaction, while the spin-lattice coupling is vital for its stability.

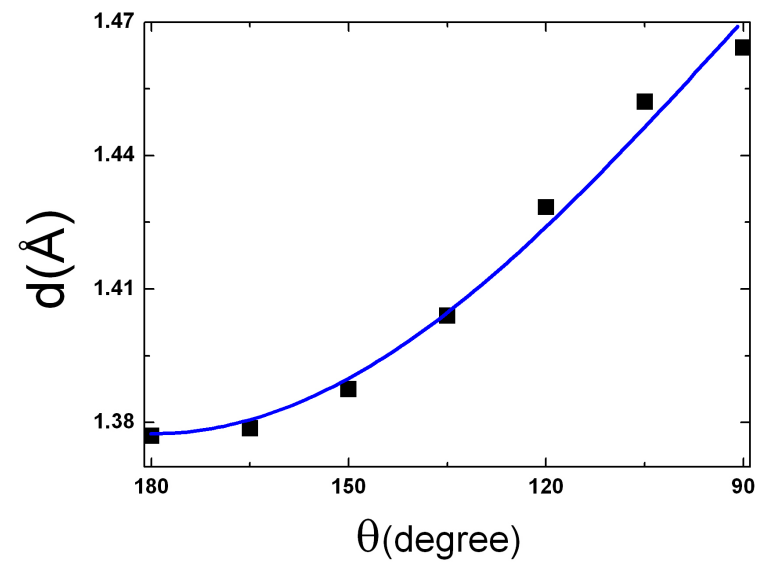

Figure 2: (color online) $d-\theta$ relation of the total energy minimum state for given spin angle $\theta$. The black squares are calculated using DFT, and the blue line is the fitted line using equation $d=d_{0}+u=d_{0}+u_{0} \cos \theta$, where the fitting parameters $d_{0}=1.470 \AA$ and $u_{0}=0.093 \AA$.

Besides the all-organic ferromagnetic ground state mentioned above, we find that a large spin-split band gap and the $100 \%$ spin polarization near the Fermi energy are specific characters of TMM and $\mathrm{CH}_{2}$ terminated ZGNR. Fig $[3$ and b respectively plot their spin-resolved band structures and density of states (DOS). From these figures, TMM and $\mathrm{CH}_{2}$ terminated ZGNRs exhibit the 
following similar features: 1) both of the spin up and down subbands manifest semiconductor character with energy gaps $\sim 1 \mathrm{eV}$; and 2 ) the spin splitting between up and down subbands near the Fermi energy $\left(E_{\mathrm{F}}\right)$ is about $0.5 \mathrm{eV}$, which induces a gapped spin-split DOS with $100 \%$ spin polarization within a wide energy region near $E_{\mathrm{F}}$. The highest occupied and lowest unoccupied energy level are proved to be extended states which can serve a large current in graphene-based transistors. In Fig 3 , the local density of states (LDOS, with energy region $E_{\mathrm{F}} \rightarrow E_{\mathrm{F}}-0.6 \mathrm{eV}$ ) of the highest (spin up) occupied energy level of $\mathrm{CH}_{2}$ terminated ZGNR is shown. Towards the practical transistors, those features provide well-defined conducting ON- and insulating OFF-states, and also the high spin-polarized current at the ON-state. By applying a positive or negative gate voltage, one can selectively shift the (purely spin up) occupied highest energy level or the lowest (purely spin down) unoccupied one towards the Fermi energy. A 100\% spin polarized current is thus produced via the gate voltage control.

For comparison, we make some comments on the nonmagnetic side group terminated ZGNRs in the literature[12]. For those materials, the sizable energy gap and $100 \%$ spin polarization in large energy range near $E_{\mathrm{F}}$ can not obtained simultaneously. For example, in the $\mathrm{NH}_{2}, \mathrm{OH}$, and $\mathrm{COOH}$ terminated ZGNRs, the energy gap is about $0.3 \mathrm{eV}$, but the spin splitting of the states near the $E_{\mathrm{F}}$ is very small. In the $\mathrm{NO}_{2}$ terminated ZGNR, although the spin splitting is relatively enlarged, the energy gap reduced to about $0.1 \mathrm{eV}$, too small to be used in transistors. Therefore, the present design has an advantage in realizing a large energy gap and a high spin polarization near the Fermi energy simultaneously.

In spintronics, the spin polarization $P(E)$ may be defined as

$$
P(E)=\frac{D O S_{\uparrow}(E)-D O S_{\downarrow}(E)}{D O S_{\uparrow}(E)+D O S_{\downarrow}(E)},
$$

which can be tuned by changing the spin polarized DOS near $E_{\mathrm{F}}$. For this purpose, we study the evolution of the spin polarization near $E_{\mathrm{F}}$ with manually varying the spin angle $\theta$ in $\mathrm{CH}_{2}$ terminated ZGNR. The result is demonstrated in Fig. 3d. As mentioned above, the DOS above and below the $E_{\mathrm{F}}$ are both $100 \%$ spin polarized in a wide energy range at $\theta=\pi$. However, as $\theta$ decreases towards $\pi / 2$, the high spin polarized region near $E_{\mathrm{F}}$ shrinks and even almost disappears at $\theta=\pi / 2$. As seen in the figure, the large value of DOS near $E_{\mathrm{F}}$ during radical rotation can keep the ON-state under certain gate voltage. Thus, controlling the exchange coupling strength by radical rotation is an effective way to tune the spin polarization.

Finally, let us comment on the possibility of taking Lieb's theorem on the repulsive Hubbard model [24] as the mechanism of the gapped ferromagnetic state, for the reason that its deduced Klein's edge [25] has been applied to explain the ferromagnetic state of some other edge ter-
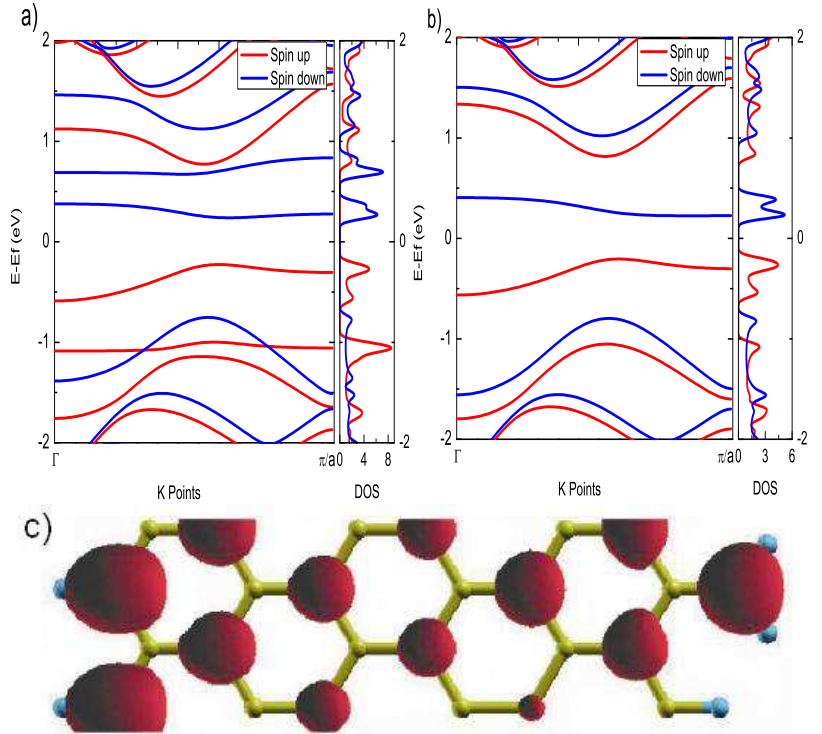

d)
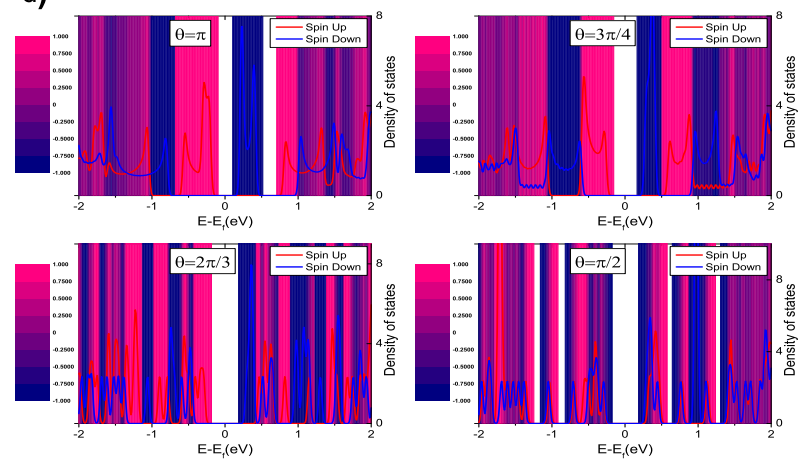

Figure 3: (color online) (a)(b) Spin-resolved band structure (left panel) and density of states (right panel) of the ground state of TMM and $\mathrm{CH}_{2}$ terminated ZGNR, respectively. (c) Local density of states(LDOS) of the ground state of $\mathrm{CH}_{2}$ terminated ZGNR. Red for up spin. (d) Spin-resolved DOS of $\mathrm{CH}_{2}$ terminated ZGNRs with spin angle $\theta$ equal to $\pi, 3 \pi / 4$, $2 \pi / 3$, and $\pi / 2$ respectively. The shaded area presents the spin polarization $\mathrm{P}(\mathrm{E})$ defined in Eq. (5).

minated GNRs in literatures [7, 26]. The central point of the Lieb's theorem is that the ground state of a bipartite lattice and half-filled band has a net total spin $S$ providing the two sublattice have different number of sites. By carefully comparing our results with Lieb's theorem, we conclude that it can not consistently explain all the features of the ground state here. The main discrepancies are summarized as follows: 1) it is the strong spin-lattice interaction, rather than the Hubbard (electron-electron) correlation, stabilizes the ground state of the present system; 2) the spatial spin order of the ground state is beyond Lieb's theorem that can only deduce a nonzero total spin; and 3) our DFT and model calculations have both omitted the quantum fluctuation of electron-electron interaction which is the curial part of Hubbard model and Lieb's theorem. Relative to Lieb's theorem, our mech- 
anism of the antiferromagnetic exchange together with strong spin-lattice interactions can explain all of the features of the gapped ferromagnetic state in a consistent manner, as already demonstrated above.

Summary.-In summary, we designed a graphenebased all-organic ferromagnetic semiconductor by terminating ZGNR with organic mangets. A large spin-split gap with $100 \%$ spin polarized DOS near $E_{\mathrm{F}}$ is obtained, which is of potential usage in spintronic devices. Combining first-principles calculations with basic model analysis, we conclude that the mechanism for the gapped ferromagnetic state is the antiferromagnetic $\pi-\pi$ exchange together with strong spin-lattice interactions between organic magnet and graphene nanoribbons. By controlling the $\pi-\pi$ interaction strength, the spin polarization of the DOS near $E_{\mathrm{F}}$ can be tuned from $100 \%$ to nearly zero. The fundamental physics makes it possible to optimally select the organic magnet towards practical applications.

The authors thank Prof. Shimin Hou of Peking University and Prof. Mingwen Zhao of Shandong University for the insightful discussions and suggestions. Support from National Basic Research Program of China (Grant No.s 2007CB925001, 2009CB929204 and 2010CB923402) and NSFC of China (Grant No.s 10874100 and 11074303) are gratefully acknowledged. Part of the computer time is supported by Physics Laboratory for High Performance Computing, Renmin University of China.

* Electronic address: wjh@ruc.edu.cn

$\dagger$ Electronic address: xsj@sdu.edu.cn

[1] K. S. Novoselov, A. K. Geim, S. V. Morozov, D. Jiang, Y. Zhang, S. V. Dubonos, I. V. Grigorieva, and A. A. Firsov, Science 306, 666 (2004)

[2] F. Schwierz, Nature Nanotechnology 5, 487 (2010).

[3] L. Yang, C. H. Park, Y. W. Son, M. L. Cohen, and S. G. Louie, Phys. Rev. Lett. 99, 186801 (2007).

[4] M. Y. Han, B. Özyilmaz, Y. B. Zhang, and P. Kim, Phys. Rev. Lett. 98, 206805 (2007).

[5] X. Li, X. Wang, L. Zhang, S. Lee, and H. Dai, Science 319, 1229 (2008).
[6] Z. Chen, Y. M. Lin, M. J. Rooks, and Ph. Avouris, Physica E 40, 228 (2007).

[7] M. Fujita, K. Wakabayashi, K. Nakada, and K. Kusakabe, J. Phys. Soc. Jpn. 65, 1920 (1996).

[8] S. Okada and A. Oshiyama, Phys. Rev. Lett. 87, 146803 (2001).

[9] Y. W. Son, M. L. Cohen, and S. G. Louie, Nature(London) 444, 347 (2006) .

[10] X. H. Zheng, R. N. Wang, L. L. Song, Z. X. Dai, X. L. Wang, and Z. Zeng Appl. Phys. Lett. 95, 123109, (2009).

[11] E. Kan, Z. Li, J. Yang, and J. G. Hou J. Am. Chem. Soc. 130, 4225, (2008).

[12] F. Cervantes-Sodi, G. Csányi, S. Piscanec, and A. C. Ferrari Phys. Rev. B 77, 165427 (2008)

[13] T. Jungwirth, J. Sinova, J. Mašek, J. Kučera, and A. H. MacDonald, Rev. Mod. Phys. 78, 809 (2006) .

[14] S. Datta, and B. Das, App. Phys. Lett. 56, 665 (1990).

[15] T. Sugawara and M. M. Matsushitta, J. Mater. Chem. 19, 1738 (2009) .

[16] N.Gorjizadeh, A. A. Farajian, K. Esfarjani, and Y. Kawazoe, Phys. Rev. B 78, 155427 (2008).

[17] V. Rigo, T. Martins, A. Silva, A. Fazzio, and R. Miwa, Phys. Rev. B 79, 075435, (2009).

[18] R. J. Baseman, D. W. Pratt, M. Chow and P. Dowd, J. Am. Chem. Soc. 98, 5726, (1976); W. T. Borden, H. Iwamura, and J. A. Berson, Acc. Chem. Res. 27, 109 (1994); D. Feller, W. T. Borden, and E. R. Davidson, J. Chem. Phys. 74, 2256 (1981); P. G. Wenthold, J. Hu, R. R. Squires, and W. C. Lineberger, J. Am. Chem. Soc. 118, 475 (1996).

[19] J. M. Soler, E. Artacho, J. D. Gale, A. García, J. Junquera, P. Ordejoón, and D. Sánchez-Portal, J. Phys.: Condens. Matter 14, 2745 (2002).

[20] J. P. Perdew, K. Burke and M. Ernzerhof, Phys. Rev. Lett. 77, 3865 (1996).

[21] P. Giannozzi et al., http://www.quantum-espresso.org.

[22] J. Jung, T. Pereg-Barnea, and A. H. MacDonald, Phys. Rev. Lett. 102, 227205 (2009).

[23] N. Atodiresei, J. Brede, P. Lazić,V. Caciuc, G. Hoffmann, R. Wiesendanger, and S. Blügel, Phys. Rev. Lett. 105, 066601 (2010).

[24] E. Lieb, Phys. Rev. Lett. 62, 1201 (1989).

[25] D. J. Klein, Chem. Phys. Lett. 217, 261 (1994).

[26] S. Lakshmi, S. Roche, and G. Cuniberti, Phys. Rev. B 80, 193404, (2009). 